Mato Vázquez, María Dorinda; Espiñeira Bellón, Eva; Chao Fernández, Rocío (2014). Dimensión afectiva hacia la matemática: resultados de un análisis en educación primaria. Revista de Investigación Educativa, 32 (1), 57-72. http://dx.doi.org/10.6018/rie.32.1.164921

\title{
DIMENSIÓN AFECTIVA HACIA LA MATEMÁTICA: RESULTADOS DE UN ANÁLISIS EN EDUCACIÓN PRIMARIA
}

\author{
María Dorinda Mato Vázquez, Eva Espiñeira Bellón y Rocío Chao Fernández \\ Universidad de A Coruña
}

\section{RESUMEN}

Este artículo evalúa la dimensión afectiva de 1180 alumnos de Educación Primaria respecto a las matemáticas durante el curso 2011-2012. Para ello se describe, en primer lugar, la percepción que tienen los estudiantes sobre las actitudes de su profesor de matemáticas, la satisfacción que sienten hacia la materia y el valor que le otorga de cara a su futuro profesional. Posteriormente se analizan las asociaciones e influencias respecto a las variables centro (público y concertado) y curso $\left(3^{\circ}, 4^{\circ}, 5^{\circ}\right.$ y $\left.6^{\circ}\right)$ mediante la prueba no paramétrica de Kruskal-Wallis. Finalmente, para conocer los efectos generales y específicos de la variable actitud sobre el rendimiento académico recurrimos a la correlación de Pearson. Finaliza el artículo con los resultados y la presentación de las conclusiones obtenidas. Podemos decir que los procesos afectivos influyen en las experiencias de los alumnos cuándo se enfrentan al aprendizaje matemático y repercuten en su calificación.

Palabras clave: Actitudes hacia las matemáticas; evaluación; fiabilidad; rendimiento.

\footnotetext{
Correspondencia:

María Dorinda Mato Vázquez. Universidad de A Coruña. Facultad de Ciencias de la Educación. Campus de Elvira, 15071 A Coruña. Teléfono: 981167000-1750

E-mail: m.matov@udc.es
} 


\section{AFFECTIVE DIMENSION TOWARDS MATHEMATICS: RESULTS OF A STUDY IN PRIMARY EDUCATION}

\section{ABSTRACT}

This article analyzed the affective dimension towards mathematics of 1,180 primary education undergraduate students during the 2011-2012 academic year. We described students' perception of their mathematics teacher's attitude, students' satisfaction with the subject matter, and to what extent students consider subject matter important for the future career. We then analyzed the influence of variables such as school type (state and state-subsidised) and school year $\left(3^{\text {rd }}, 4^{\text {th }}, 5^{\text {th }}\right.$, and $6^{\text {th }}$ grade) using non-parametric Kruskal-Wallis test. We used Pearson's correlations to analyze the general and specific effects of the attitude on academic performance variable. The results showed that affective processes do influence students' experience of learning mathematics as well as their assessment results.

Keywords: attitudes towards the mathematics; evaluation; reliability; academic performance.

\section{INTRODUCCIÓN}

Los profesionales de la enseñanza centramos, cada vez más, nuestra atención en las barreras a las que se enfrentan los estudiantes mientras aprenden. El rendimiento académico, los altos índices de suspensos, las elevadas tasas de abandono en las enseñanzas medias y superior son motivos que han preocupado a profesores de diferentes niveles educativos y a los mismos alumnos (Abalde, Barca, Muñoz y Ziemer, 2009).

En la Universidad de A Coruña, las investigaciones llevadas a cabo por los docentes del Área de Didáctica de la Matemática con alumnos de Enseñanza Obligatoria, reflejan un rendimiento y unas actitudes deficientes en esta asignatura; peores conforme avanzan de curso (Mato, 2006).

Es verdad que el concepto de rendimiento académico es multidimensional; no podemos, solamente, valorar dentro del proceso educativo la productividad del alumno sino tener en cuenta otros elementos de orden personal e instrumental que contribuyen a un buen resultado en educación (Peralbo y Barca, 2003) y que, entre los procesos de aprendizaje, rendimiento, éxito y fracaso escolares existe una relación intrínseca Abramowicz y Moll (2000). También es cierto que la afectividad ha adquirido, a lo largo del tiempo, varias acepciones y definiciones y debe ser abordada con una significación amplia, refiriéndonos a las vivencias de los individuos y a las formas de expresión más complejas y, esencialmente humanas, como respuestas positivas o negativas, producidas durante el proceso de aprendizaje. Y en ese proceso, las manifestaciones del profesor pueden influir tanto en el aprendizaje como en la enseñanza de la matemática ya que las razones de la impopularidad de esta disciplina son variadas, pero como sostiene Martínez Padrón (2005), responden sin duda, tanto a aspectos cognitivos como afectivos. Más aún, muchas veces este desagrado está ligado con rendimientos bajos y éstos a su vez con el fracaso escolar de los estudiantes, el cual no siempre se corresponde con indicadores de bajo desarrollo cognitivo.

En referencia al desarrollo intelectual, las aportaciones de Piaget (1981) van en la misma línea; el autor considera que es un proceso que comprende un aspecto cogni- 
tivo y un aspecto afectivo. Afirma que vida afectiva y vida cognitiva son inseparables, porque todo intercambio con el medio presupone, al mismo tiempo, estructuración y valorización. Asimismo, añade que no se podría razonar -incluso en matemática- sin experimentar ciertos sentimientos, mientras que tampoco existen afecciones sin un mínimo de comprensión. El acto de la inteligencia presupone, pues, una regulación energética interna.

Nuestra experiencia como profesores y las investigaciones realizadas con alumnos de Secundaria (Muñoz y Mato, 2008), constatan cómo muchos estudiantes están bloqueados y aterrorizados ante las matemáticas. Algunos tienen crisis de ansiedad y pánico, y un temor que a menudo dura toda la vida tomando un lugar permanente si no se hace nada por evitarlo.

Tomando como base las investigaciones y experiencias que se centran en los mecanismos afectivos en el desarrollo de actitudes hacia la asignatura de matemáticas, podemos destacar las de Gómez Chacón (2000) o Blanco (2008). Estos autores especifican tres descriptores básicos del dominio afectivo, (emociones, actitudes y creencias) y consideran de gran relevancia las repercusiones que tienen en su aprendizaje.

En relación a las actitudes Estrada (2002) las define como construcciones teóricas que se infieren de ciertos comportamientos externos, y Callejo (2004) dice que son, predisposiciones estables a valorar y a actuar, que se basan en una organización relativamente duradera de creencias en torno a la realidad que predispone a actuar de determinada forma o respuestas positivas o negativas, producidas durante el proceso de aprendizaje (Ashcraft, 2002).

En general, las actitudes son adquiridas (Zabalza, 1994) y ocupan un lugar central, tanto en la construcción de la persona como en el conocimiento (Zan, Brown, Evans, y Hannula, 2006).

Las actitudes hacia las matemáticas surgen desde edades muy tempranas $y$, aunque tienden a ser favorables en un principio, disminuyen a medida que avanzan escolarmente (Aliaga y Pecho, 2000) y se produce un descenso significativo en su utilidad de cara al futuro (Watt, 2000 y Broc Cavero, 2006).

En cuanto a las creencias Gil, Blanco y Guerrero (2005) las define como verdades personales indiscutibles, sustentadas por cada uno, derivadas de la experiencia o de la fantasía, que tiene un fuerte componente evaluativo y afectivo y Gómez-Chacón (2002) las considera estructuras cognitivas que permiten al individuo organizar y filtrar las informaciones recibidas y que van construyendo su noción de realidad y su visión del mundo.

Por consiguiente, el profesor de Matemáticas debe conocer cómo y quienes resuelven los problemas, cómo seleccionan los procedimientos y los factores que facilitan o dificultan el aprendizaje (Gómez-Chacón, 2007)

Hoy la enseñanza aboga por un mayor protagonismo del alumno, actor en la construcción del conocimiento. Es aquí donde se forman las creencias sobre la matemática, su enseñanza y su aprendizaje. Murillo Torrecilla, y Hernández Castilla (2011) consideran las creencias hacia los profesores como una de las variables que incide en su práctica educativa, ya que la conducta del profesor guía el sistema personal de creencias y valores. 
En relación a las emociones Gómez-Chacón (2000) señala que surgen en respuesta a un suceso, interno o externo, que tiene una carga de significado positiva o negativa para el individuo.

Para Gómez Chacón (2006), la competencia emocional constituye una meta-habilidad que determina el grado de destreza que alcanzaremos en el dominio de todas nuestras facultades (entre las cuales se incluye el intelecto puro).

Los datos aportados por los estudios de Woodard (2004), permiten reflexionar sobre la relación que se establece entre las actitudes, creencias y emociones. Por una parte, la experiencia que tiene el estudiante al aprender matemáticas influye en la formación de sus creencias, éstas tienen una consecuencia directa en su comportamiento y el resultado es la reacción emocional positiva o negativa en las situaciones de aprendizaje y en su capacidad para aprender.

Por otra parte Torre y Mato (2009) exponen que las actitudes negativas aumentan durante los primeros cursos de Educación Secundaria, alcanzando su cumbre en tercero y cuarto y se estabiliza durante los cursos de Bachillerato. También Fierro-Hernández (2006) y Broc (2006) señalan que si los profesores muestran interés en su trabajo, se producen mejoras significativas en las actitudes de los estudiantes. Mato (2006) llevó a cabo un estudio para analizar las actitudes de 1220 alumnos de Educación Secundaria Obligatoria pertenecientes a siete centros (586 chicos y 634 chicas). Los resultados concluyen que las actitudes hacia la matemática varían en función del tipo de centro (público o concertado) y del curso $\left(1^{\circ}, 2^{\circ}, 3^{\circ}\right.$ y $\left.4^{\circ}\right)$. Así mismo existe una alta correlación positiva y significativa entre la actitud y el rendimiento.

Asimismo, Morales (2006) comprueba la incidencia de las actitudes en el rendimiento en estudiantes de Educación Primaria y Kazelskis (2000) lleva a cabo una investigación con tres grupos de cursos escolares (de $4^{\circ}$ a $6^{\circ}$ de Educación Primaria, $1^{\mathrm{o}}$ y $2^{\mathrm{o}}$ de Educación Secundaria y $1^{\mathrm{o}}$ y $2^{\mathrm{o}}$ de Bachillerato) y asegura que la relación entre las actitudes hacia las matemáticas y el éxito matemático es significativo desde el $4^{\circ}$ curso de Educación Primaria.

Por tanto no deja de ser alarmante, siendo la matemática una de las asignaturas más importantes del currículum, es a la vez, de las más temidas (Tyson, 2001) y la que tiene un rendimiento más deficiente (Bazán y Aparicio, 2006).

Es habitual que los profesores nos encontremos con alumnos que reaccionan emocionalmente de forma negativa a la hora de trabajar con números, problemas, exámenes o salir al encerado; estudiantes incapaces de aprender matemáticas y con sentimientos de culpabilidad por su fracaso; falsos mitos de que la matemática es para gente prestigiosa, muy inteligente y creativa; dificultades para pensar por ellos mismos; suponer que la autoridad y la única verdad es la del profesor y la del libro de texto y finalmente, alumnos que se dan por vencidos, se resignan a no aprender creyendo que no son capaces, carentes de autoestima matemática. Las creencias que tiene un estudiante acerca de sí mismo y acerca de las matemáticas, y las veces que en su vida se ha repetido esa situación pueden dar lugar a reacciones de bloqueo irreversibles.

En este escenario emocional si en algún momento no se resuelven los obstáculos que una persona tiene ante la matemática, el pánico puede llegar a automatizarse y originar actitudes negativas que le lleven al fracaso e incluso dudar de su capacidad 
intelectual. Son estudiantes que consideran sus esfuerzos inútiles, manifiestan sentimientos de indefensión o pasividad en la construcción de su persona, así como en el conocimiento, lo que delimitará su rendimiento y su futuro profesional (Blanco, 2008).

En definitiva, la falta de implicación de un estudiante en el proceso de enseñanza y aprendizaje repercute directamente en su esfuerzo personal en relación con esta materia, en la motivación y el autoconcepto (Mato y Muñoz, 2010).

A tenor de lo expuesto, los profesores de matemáticas debemos tener una idea general de los afectos de nuestros alumnos con relación a la asignatura y asegurarnos de que poseen una base sólida que fomente actitudes positivas (Gil, Blanco y Guerrero, 2005), desarrolle creencias efectivas para el aprendizaje y desenvuelva emociones reales hacia la matemáticas. De ese modo, el alumno tendrá una y predisposición favorable, se creerá capaz de entenderlas y hacer uso de la asignatura en su vida cotidiana (Muñoz y Mato, 2008).

\section{OBJETIVO}

El objetivo principal que se persigue con esta investigación es analizar las actitudes que presentan los alumnos del $2^{\circ}$ y $3^{\circ}$ ciclo de Educación Primaria en relación a la matemática.

Este objetivo general se desdobla en los siguientes objetivos específicos:

- Descubrir la percepción que tienen los estudiantes acerca de su profesor de matemáticas.

- Conocer la motivación, utilidad y satisfacción que sienten al resolver matemáticas.

- Analizar si hay diferencias en las actitudes de los alumnos hacia las matemáticas respecto al centro y al curso.

- Averiguar si las actitudes influyen en el rendimiento.

\section{HIPÓTESIS}

H1: La actitud hacia las matemáticas se ve influenciada por variables como el centro, el curso y el sexo.

H2: El rendimiento en matemáticas se ve influenciado por las actitudes.

\section{MUESTRA}

En este estudio han participado 10 centros educativos, 5 públicos y 5 privados de enseñanza Primaria del municipio de A Coruña, que, en el plazo solicitado, accedieron a participar en el estudio durante el curso 2011-12.

Los colegios se eligen al azar para no condicionar los resultados, pero con una representación proporcional de todos los centros públicos y concertados, (en el momento de pasar los cuestionarios existían en A Coruña 17 centros públicos y 17 centros concertados). Evitamos los colegios con rendimiento académico extremo. Se prescinde, por ejemplo, de las unidades de educación especial.

La distribución de la muestra respecto al curso y al tipo de centros se indican en la Tabla 1. 
TABLA 1

CLASIFICACIÓN DE LA MUESTRA SEGÚN CENTRO Y CURSO

\begin{tabular}{lll}
\hline Centro & Frecuencia & Porcentaje \\
\hline Público & 524 & 44,4 \\
\hline Concertado & 656 & 55,6 \\
\hline Curso & Frecuencia & Porcentaje \\
\hline Tercero & 325 & 27,5 \\
\hline Cuarto & 307 & 26,0 \\
\hline Quinto & 249 & 21,1 \\
\hline Sexto & 299 & 25,3 \\
\hline 1180 & 100,0 & \\
\hline Total & $\mathbf{1 1 8 0}$ & $\mathbf{1 0 0 , 0}$ \\
\hline
\end{tabular}

Para medir el rendimiento elegimos la calificación que obtuvieron los alumnos en matemáticas en el curso anterior: suspenso, aprobado, bien, notable, sobresaliente (Tabla 2).

TABLA 2

DISTRIBUCIÓN DE LA MUESTRA POR CALIFICACIÓN

\begin{tabular}{lllll}
\hline & Frecuencia & Porcentaje & Porcentaje válido & Porcentaje acumulado \\
\hline Suspenso & 196 & 16,6 & 16,6 & 16,6 \\
\hline Aprobado & 155 & 13,1 & 13,1 & 29,7 \\
\hline Bien & 176 & 14,9 & 14,9 & 44,7 \\
\hline Notable & 514 & 43,6 & 43,6 & 88,2 \\
\hline Sobresaliente & 139 & 11,8 & 11,8 & 100,0 \\
\hline Total & 1180 & 100,0 & 100,0 & \\
\hline
\end{tabular}

\section{INSTRUMENTO DE MEDIDA}

El instrumento utilizado para la investigación fue elaborado por una de las investigadoras del estudio (Mato, 2006), a partir de la Escala de actitudes de Fennema y Sherman (1976) para una muestra de 1220 estudiantes de Educación Secundaria. Es una escala de 19 ítems distribuidos en dos factores "la actitud del profesor percibida por el alumno" y "agrado y utilidad de las matemáticas en el futuro", con un coeficiente de fiabilidad Alpha de Cronbach de 9,706 y una validez de constructo 9,772. 
Solicitamos la colaboración de distintos profesores que imparten matemáticas en los cursos investigados para que analizaran los ítems e hicieran las modificaciones oportunas.

Su opinión fue esencial para conocer si los alumnos serían capaces de contestar con facilidad y sin confusiones.

Los criterios de selección estuvieron fundamentados en cada uno de los aspectos de coherencia, representatividad y calidad técnica. Una vez obtenidas las puntuaciones de los jueces, únicamente se realizaron cambios referidos a la redacción de un ítem: “En primaria me gustaban las matemáticas" por "En los cursos anteriores me gustaban las matemáticas" (ítem 11).

Hechas las modificaciones, se realiza el análisis de la fiabilidad alfa de Cronbach obteniendo un resultado muy aceptable de ,8879 y una validez de constructo de ,932.

Se aplica el cuestionario definitivo a la muestra final en diciembre del curso 2011-12.

\section{Interpretación de los factores del cuestionario}

Factor I: La actitud del profesor percibida por el alumno. Describe la percepción que tienen los estudiantes sobre las actitudes de su profesor de matemáticas. Hace referencia al trato que tiene el profesor con sus alumnos, cómo los anima, si se divierte en clase, cómo logra que les interesen las matemáticas, cómo son las clases (si son participativas).

Incluye los siguientes ítems:

- El profesor me anima para que estudie más matemáticas (2)

- El profesor me aconseja y me enseña a estudiar (3)

- Me siento motivado en clase de matemáticas (5)

- El profesor se divierte cuando nos enseña matemáticas (6)

- Pregunto al profesor cuando no entiendo algún ejercicio ( 7)

- El profesor de matemáticas me hace sentir que puedo ser bueno en matemáticas (9)

- El profesor tiene en cuenta los intereses de los alumnos ( 10)

- Me gusta cómo enseña mi profesor de matemáticas (12)

- Después de cada evaluación, el profesor me comenta los progresos hechos y las dificultades encontradas (14)

- El profesor se interesa por ayudarme a solucionar mis dificultades con las matemáticas (15)

- En general, las clases son participativas (19)

Factor II: Agrado y utilidad de las matemáticas en el futuro. Se refiere a la satisfacción que siente el estudiante, la confianza que tiene en sí mismo, el valor que les otorga a las matemáticas de cara al futuro, la utilidad tanto desde el punto de vista racional y cognitivo como desde la perspectiva afectiva y comportamental.

Incluye los siguientes ítems:

- Las matemáticas serán importantes para mi profesión (1)

- Las matemáticas son útiles para la vida cotidiana (4) 
- Entiendo los ejercicios que me manda el profesor para resolver en casa (8)

- En los cursos anteriores me gustaban las matemáticas (11)

- Espero utilizar las matemáticas cuando termine de estudiar (13)

- Saber matemáticas me ayudará a ganarme la vida (16)

- Soy bueno en matemáticas (17)

- Me gustan las matemáticas (18)

\section{APLICACIÓN DEL CUESTIONARIO}

Los cuestionarios fueron aplicados, durante el curso 2011-2012. La puesta en contacto con los colegios se hace en un primer momento telefónicamente con cada uno de los directores de los centros. Posteriormente hay una entrevista en la que participan el orientador y el investigador y se explican los motivos y de qué consta la investigación. Se pide que sea el orientador del centro el que aplique los cuestionarios, de forma colectiva, solicitando que no estuviera presente el profesor de matemáticas en el aula con el fin de no mediatizar las respuestas de los alumnos.

No se impuso limitación de tiempo y elegimos el horario lectivo para evitar esa desgana manifiesta que, generalmente, se tiene a contestar encuestas. Se insiste en la sinceridad y concentración a la hora de responder los ítems.

Inicialmente se lleva a cabo una prueba piloto compuesta por 20 alumnos de quinto de Primaria con características idénticas a las que posteriormente constituirían la muestra final pero que no formarían parte de ella.

\section{PROCEDIMIENTOSYTÉCNICAS DE ANÁLISIS}

Con respecto a la realización de los cálculos y al tratamiento estadístico general de los datos se utilizó el grupo de programas SPSS, en su versión 15.0.

Analizamos las asociaciones e influencias entre las variables mediante análisis de las diferencias que presentan teniendo en cuenta otras variables como centro y curso. Utilizamos la prueba no paramétrica de Kruskal-Wallis para analizar las diferencias en el caso del curso y del centro, y, para conocer los efectos generales y específicos de la variable actitud sobre el rendimiento académico utilizamos la correlación de Pearson.

\section{ANÁLISIS DESCRIPTIVOS}

Coincidiendo con los objetivos de la investigación formulados, el análisis de los datos se ha estructurado en dos apartados. En primer lugar evaluamos los resultados del cuestionario en relación a la percepción que tienen los estudiantes sobre las actitudes de su profesor de matemáticas y, en segundo lugar analizamos los resultados que tienen que ver con el agrado, utilidad y satisfacción hacia el estudio de las matemáticas.

El plan de análisis fue el siguiente:

Previamente realizamos un análisis descriptivo de los ítems que nos ayudan a contextualizar las muestras referentes a: "La actitud del profesor percibida por los alumnos" y "Agrado y utilidad de las matemáticas en el futuro". 


\section{Resultados del factor “La actitud del profesor percibida por los alumnos”}

Comprende los siguientes ítems: 2, 3, 5, 6, 7, 9, 10, 12, 14, 15 e 19.

Cabe destacar que la mayoría de los alumnos responden mucho o bastante a este factor; en referencia al hecho de que el profesor les anima, les aconseja y les enseña, les comenta los progresos hechos y las dificultades encontradas, tiene en cuenta sus intereses, le preguntan cuándo no entienden algún ejercicio, les gusta cómo enseña, las clases son participativas y se sienten motivados en clase de matemáticas.

Los datos de la Tabla siguiente resumen y clarifican todos los resultados en porcentajes.

TABLA 3

RESULTADOS DEL FACTOR "LA ACTITUD DEL PROFESOR PERCIBIDA POR LOS ALUMNOS"

\begin{tabular}{|c|c|c|c|c|c|c|c|c|c|c|c|}
\hline & $\begin{array}{c}\text { ÍTEM } \\
2 \\
\end{array}$ & $\begin{array}{c}\text { ÍTEM } \\
3 \\
\end{array}$ & $\begin{array}{c}\text { ÍTEM } \\
5 \\
\end{array}$ & $\begin{array}{c}\text { ÍTEM } \\
6 \\
\end{array}$ & $\begin{array}{c}\text { ÍTEM } \\
7 \\
\end{array}$ & $\begin{array}{c}\text { ÍTEM } \\
9 \\
\end{array}$ & $\begin{array}{c}\text { ÍTEM } \\
10 \\
\end{array}$ & $\begin{array}{c}\text { ÍTEM } \\
12 \\
\end{array}$ & $\begin{array}{c}\text { ÍTEM } \\
14 \\
\end{array}$ & $\begin{array}{c}\text { ÍTEM } \\
15 \\
\end{array}$ & $\begin{array}{c}\text { ÍTEM } \\
19 \\
\end{array}$ \\
\hline Nada & , 3 & 3,2 &, 3 & 3,6 & 3,3 & 0,3 & 4,5 & ,3 & 3,4 & 3,4 & 3,4 \\
\hline Un poco & 11,3 & 8,6 & 10,4 & 18,5 & 8,5 & 9,6 & 16,9 & 9,9 & 8,4 & 8,4 & 8,4 \\
\hline Regular & 14,7 & 25,0 & 13,6 & 18,8 & 24,9 & 15,9 & 19,2 & 16,0 & 25,0 & 25,0 & 25,0 \\
\hline Bastante & 31,5 & 27,7 & 32,4 & 29,2 & 26,1 & 31,6 & 28,7 & 31,5 & 26,3 & 26,0 & 26,0 \\
\hline Mucho & 42,3 & 35,4 & 43,2 & 29,9 & 37,2 & 42,5 & 30,7 & 42,2 & 36,9 & 37,2 & 37,2 \\
\hline Total & 100,0 & 100,0 & 100,0 & 100,0 & 100,0 & 100,0 & 100,0 & 100,0 & 100,0 & 3,4 & 100,0 \\
\hline
\end{tabular}

\section{Resultados del factor “Agrado y utilidad de las matemáticas en el futuro”}

Comprende los siguientes ítems: 1, 4, 8, 11, 13, 16, 17, 18 .

Debemos destacar que, en general, se obtienen valoraciones altas al considerar que las matemáticas serán importantes para su profesión, son útiles para la vida, entienden los ejercicios que les manda el profesor para resolver en casa, en los cursos anteriores les gustaban, esperan utilizarlas cuando terminen de estudiar, se consideran buenos y les gustan las matemáticas.

TABLA 4

RESULTADOS DEL FACTOR “AGRADO Y UTILIDAD DE LAS MATEMÁTICAS EN EL FUTURO"

\begin{tabular}{llllllllll} 
& ÍTEM 1 & ÍTEM 4 & ÍTEM 8 & ÍTEM 11 & ÍTEM 13 & ÍTEM 16 & ÍTEM 17 & ÍTEM 18 \\
\hline Nada & 3,3 & 3,5 & 3,4 & 4,5 & 3,3 & 3,4 & 3,4 & 4,3 \\
\hline Un poco & 8,6 & 8,5 & 8,6 & 15,8 & 8,4 & 8,2 & 8,2 & 8,3 \\
\hline Regular & 24,9 & 24,7 & 24,5 & 22,4 & 24,9 & 25,3 & 25,2 & 25,0 \\
\hline
\end{tabular}




\begin{tabular}{lllllllll} 
& ÍTEM 1 & ÍTEM 4 & ÍTEM 8 & ÍTEM 11 & ÍTEM 13 & ÍTEM 16 & ÍTEM 17 & ÍTEM 18 \\
\hline Bastante & 27,6 & 27,8 & 26,3 & 25,2 & 26,4 & 26,0 & 26,1 & 26,5 \\
\hline Mucho & 35,5 & 35,5 & 37,2 & 32,1 & 36,9 & 37,1 & 37,1 & 35,8 \\
\hline Total & 100,0 & 100,0 & 100,0 & 100,0 & 100,0 & 100,0 & 100,0 & 100,0 \\
\hline
\end{tabular}

\section{ANÁLISIS DE LAACTITUD}

Se calculó la media y la desviación típica de los datos referidos a cada uno de los ítems de la variable actitud y de los dos factores en los que se agrupaban según el análisis factorial. La media de actitud total se encuadra dentro de las categorías bastante y mucho de la escala del cuestionario. Por factores, "agrado y utilidad de las matemáticas" es el valor más alto y "la actitud del profesor percibida por el alumno" el más bajo (Tabla 5).

TABLA 5

MEDIAS Y DESVIACIÓN TÍPICA DE LOS FACTORES

\begin{tabular}{lll}
\hline & Media & Desv. típ. \\
\hline Valores de los factores & & \\
\hline Media de actitud & 3,86 &, 796 \\
\hline Actitud del profesor percibida por el alumno & 3,78 &, 859 \\
\hline Agrado y utilidad de las matemáticas & 3,95 &, 877 \\
\hline
\end{tabular}

\section{ANÁLISIS DE LA ACTITUD PORTIPO DE CENTRO}

Para realizar este análisis aplicamos la prueba de Kruskal-Wallis. Los datos obtenidos muestran que no hay diferencias significativas en cada una de las dos dimensiones de la actitud (actitud del profesor percibida por el alumno $\mathrm{p}>, 05$; agrado y utilidad de las matemáticas $\mathrm{p}>, 05)$, así como en la actitud total ( $\mathrm{p}>, 05)$.

TABLA 6

PRUEBA DE KRUSKAL-WALLIS ACTITUD-TIPO DE CENTRO

\begin{tabular}{|c|c|c|c|c|c|c|}
\hline & $\begin{array}{l}\text { Centro donde } \\
\text { estudias }\end{array}$ & $\mathbf{N}$ & $\begin{array}{l}\text { Rango } \\
\text { promedio }\end{array}$ & $\begin{array}{l}\text { Chi- } \\
\text { cuadrado }\end{array}$ & Gl & $\begin{array}{l}\text { Signifi. } \\
\text { asintótica }\end{array}$ \\
\hline \multirow[t]{3}{*}{$\begin{array}{l}\text { Actitud del profesor } \\
\text { percibida por el alumno }\end{array}$} & Público & 524 & 611,57 & 13,216 & 3 & 1,004 \\
\hline & Concertado & 656 & 571,17 & & & \\
\hline & Total & 1180 & & & & \\
\hline $\begin{array}{l}\text { Agrado y utilidad } \\
\text { De las matemáticas }\end{array}$ & Público & 524 & 565,42 & 22,743 & 3 & .098 \\
\hline
\end{tabular}




\begin{tabular}{lllllll}
\hline & $\begin{array}{l}\text { Centro donde } \\
\text { estudias }\end{array}$ & N & $\begin{array}{l}\text { Rango } \\
\text { promedio }\end{array}$ & $\begin{array}{l}\text { Chi- } \\
\text { cuadrado }\end{array}$ & Gl & $\begin{array}{l}\text { Signifi. } \\
\text { asintótica }\end{array}$ \\
\hline & Concertado & 656 & 638,84 & & & \\
\hline Tctitud total & Total & 1180 & & & & 1.001 \\
\hline & Público & 524 & 574,30 & 17,507 & 3 & \\
\hline
\end{tabular}

\section{ANÁLISIS DE LA ACTITUD POR CURSO}

Para realizar este análisis procedemos de la misma manera que con la variable anterior, aplicamos la prueba no paramétrica (Kruskal-Wallis) con el fin de determinar la existencia o no de diferencias significativas en cuanto a la actitud y a sus factores.

La prueba nos presenta diferencias significativas solamente en uno de los factores de actitud (agrado y utilidad de las matemáticas $=11,277, \mathrm{p}<, 05$ ), y no en el caso de actitud del profesor percibida por el alumno $=3,228, p>, 05$; ni en la actitud total $(1,789$, $\mathrm{p}>$,05) (Tabla 7).

TABLA 7

PRUEBA DE KRUSKAL-WALLIS ACTITUD-CURSO

\begin{tabular}{|c|c|c|c|c|c|c|}
\hline & Curso & $\mathbf{N}$ & $\begin{array}{l}\text { Rango } \\
\text { promedio }\end{array}$ & $\begin{array}{l}\text { Chi- } \\
\text { cuadrado }\end{array}$ & Gl & $\begin{array}{l}\text { Signifi. } \\
\text { Asintótica }\end{array}$ \\
\hline \multirow[t]{5}{*}{$\begin{array}{l}\text { Actitud del profesor } \\
\text { percibida por el alumno }\end{array}$} & Tercero & 325 & 621,29 & 3,228 & 3 & ,358 \\
\hline & Cuarto & 307 & 604,81 & & & \\
\hline & Quinto & 249 & 585,16 & & & \\
\hline & Sexto & 299 & 631,97 & & & \\
\hline & Total & 1180 & & & & \\
\hline \multirow[t]{5}{*}{$\begin{array}{l}\text { Agrado y utilidad de las } \\
\text { matemáticas }\end{array}$} & Tercero & 325 & 599,46 & 11,277 & 3 & ,010 \\
\hline & Cuarto & 307 & 650,18 & & & \\
\hline & Quinto & 249 & 630,96 & & & \\
\hline & Sexto & 299 & 561,33 & & & \\
\hline & Total & 1180 & & & & \\
\hline \multirow[t]{5}{*}{ Actitud total } & Tercero & 325 & 610,13 & 1,789 & 3 & 617 \\
\hline & Cuarto & 307 & 632,88 & & & \\
\hline & Quinto & 249 & 602,00 & & & \\
\hline & Sexto & 299 & 597,93 & & & \\
\hline & Total & 1180 & & & & \\
\hline
\end{tabular}




\section{ANÁLISIS DE LA RELACIÓN ENTRE ACTITUD Y RENDIMIENTO EN MATEMÁTICAS}

Para proceder al contraste de esta hipótesis utilizamos, al igual que en los análisis anteriores, la prueba no paramétrica (Kruskal-Wallis). Mediante este análisis pretendemos descubrir si existen diferencias significativas en la variable actitud respecto a la calificación obtenida por los sujetos.

Los resultados son los que se muestran en la Tabla 8. Como podemos observar, a nivel general, en cuanto a las puntuaciones obtenidas en valores de actitud, encontramos que 1 a s diferencias son significativas con valores de,000. Es decir hay diferencias significativas respecto a la calificación de los estudiantes en cuanto a los factores "actitud del profesor percibida por el alumno" y "agrado y utilidad de las matemáticas".

TABLA 8

PRUEBA DE KRUSKAL-WALLIS. ACTITUD-RENDIMIENTO

\begin{tabular}{llll}
\hline & $\begin{array}{l}\text { Actitud del profesor } \\
\text { percibida por el alumno }\end{array}$ & $\begin{array}{l}\text { Agrado y utilidad de } \\
\text { las matemáticas }\end{array}$ & Actitud total \\
\hline Chi-cuadrado & 469,328 & 992,559 & 817,812 \\
\hline Gl & 4 & 4 & 4 \\
\hline Sig. asintót. &, 000 &, 000 &, 000 \\
\hline
\end{tabular}

\section{EFECTOS GENERALES DE LAVARIABLE ACTITUD SOBRE EL RENDIMIENTO}

Para analizar las asociaciones e influencias entre las variables utilizadas y los factores en que se descomponen optamos por realizar la correlación de Pearson. Los resultados muestran la existencia de correlaciones significativas entre ellas. Así observamos que la actitud y la calificación de los alumnos tiene una correlación positiva y relativamente alta y significativa $(, 791)$; es decir que los sujetos obtienen mayores calificaciones a medida que la actitud es más positiva.

TABLA 9

CORRELACIONES CALIFICACIÓN-ACTITUD

\begin{tabular}{llll}
\hline & & $\begin{array}{l}\text { Calificación de } \\
\text { los alumnos }\end{array}$ & Actitud total \\
\hline Calificación de los alumnos & Correlación de Pearson & 1 &, $791\left({ }^{* *}\right)$ \\
\hline & Sig. (bilateral) &. &, 000 \\
\hline Actitud total & Correlación de Pearson &, $791\left(^{* *}\right)$ & 1 \\
\hline & Sig. (bilateral) &, 000 &. \\
\hline & $\mathrm{N}$ & 1180 & 1180 \\
\hline
\end{tabular}

** La correlación es significativa al nivel 0,01 (bilateral). 
Los valores que presentamos a continuación representan las correlaciones existentes entre el rendimiento obtenido en matemáticas y cada uno de los factores de actitud. Los resultados muestran valores positivos y significativos en todos los casos de forma que al aumentar las calificaciones su actitud hacia el "agrado y la utilidad de las matemáticas" es mayor y también la "actitud del profesor percibida por el alumno". Consideramos altos estos valores, pues obtenemos correlaciones de ,896 entre la calificación que obtienen y la utilidad de las matemáticas y de ,754 con la actitud del profesor (Tabla 10).

TABLA 10

CORRELACIONES CALIFICACIÓN-FACTORES DE ACTITUD

\begin{tabular}{lllll}
\hline & & $\begin{array}{l}\text { Calificación } \\
\text { dos alumnos }\end{array}$ & $\begin{array}{l}\text { Agrado y } \\
\text { utilidad de las } \\
\text { matemáticas }\end{array}$ & $\begin{array}{l}\text { Actitud del } \\
\text { profesor percibida } \\
\text { por los alumnos }\end{array}$ \\
\hline $\begin{array}{l}\text { Calificación de los } \\
\text { alumnos }\end{array}$ & $\begin{array}{l}\text { Correlación de } \\
\text { Pearson }\end{array}$ & 1 &, $896\left(^{* *}\right)$ &, $754\left(^{* *}\right)$ \\
\hline & Sig. (bilateral) &. &, 000 &, 000 \\
\hline $\begin{array}{l}\text { Agrado y utilidad de las } \\
\text { matemáticas }\end{array}$ & $\begin{array}{l}\text { Correlación de } \\
\text { Pearson }\end{array}$ &, $896\left(^{* *}\right)$ & 1 &, $789\left(^{* *}\right)$ \\
\hline & Sig. (bilateral) &, 000 &. &, 000 \\
\hline $\begin{array}{l}\text { Actitud del profesor } \\
\text { percibida por los } \\
\text { alumnos }\end{array}$ & $\begin{array}{l}\text { Correlación de } \\
\text { Pearson }\end{array}$ &, $754\left(^{(* *}\right)$ &, $789\left(^{* *}\right)$ & 1 \\
\hline & Sig. (bilateral) &, 000 &, 000 &. \\
\hline & $\mathrm{N}$ & 1180 & 1180 & 1180 \\
\hline
\end{tabular}

** La correlación es significativa al nivel 0,01 (bilateral).

\section{CONCLUSIONES}

En nuestra investigación hemos tratado de estudiar el aprecio, gusto y motivación hacia la asignatura de matemáticas, así como la percepción que tienen de su profesor. También queríamos saber si hay diferencias de actitud respecto al centro y al curso. Por último, si las actitudes y el rendimiento académico correlacionan.

A tenor de lo expuesto y de los resultados encontrados, consideramos que los alumnos de la muestra tienen una percepción buena de su profesor y de las matemáticas, están motivados y se consideran buenos.

También se pone de manifiesto que, en las relaciones entre los procesos afectivos y las variables centro y curso hay diferencias únicamente en el factor "agrado y utilidad de las matemáticas" respecto a los cursos $4^{\circ}$ e $6^{\circ}$. Sin embargo no se manifiestan diferencias en el otro factor ni respecto al centro.

En cuanto a la relación actitud-rendimiento, observamos valores positivos y significativos en todos los casos de forma que aumentan las calificaciones en función de las actitudes. Estos resultados coinciden con los hallados por Bazán (2006). 
Por ello, consideramos que, a partir de los datos aportados podemos enunciar aspectos significativos sobre los afectos y el saber matemático, sobre lo que piensan los alumnos y sus profesores y aportar estrategias que contribuyan al desarrollo del aprendizaje matemático.

No podemos obviar que las actitudes positivas no sólo ayudan a explicar el rendimiento de los alumnos, sino que también son en sí mismas un resultado importante de la educación. Por lo general se considera probable que los alumnos eficaces, en el momento de cambiar de curso o dejar el centro, sigan aprendiendo a lo largo de toda su vida, especialmente aquellos que han aprendido a regular su propio aprendizaje.

Con el fin de realizar una síntesis de los resultados observados en esta investigación y en otros estudios, a grandes trazos, es posible afirmar que, con el paso de los cursos (de Primaria hasta $4^{\circ}$ de ESO), se produce un descenso de las actitudes de los alumnos ante el estudio de la matemática (Amorín, 2004). En primer lugar, resulta significativo que el interés por la matemática decrece al llegar a Secundaria, dato sorprendente si tenemos en cuenta la relevancia del conocimiento matemático de cara a las posibilidades futuras, tanto académicas como en relación a las salidas profesionales y a las demandas de la sociedad, coincidiendo con los estudios realizados por Hidalgo, Maroto y Palacios (2005).

Del mismo modo, la utilidad de las matemáticas presenta un descenso más acentuado conforme avanzan de curso. Tal vez, la razón se explique por la forma cómo se presentan las matemáticas, en muchos casos, apartadas de la vida real, descontextualizadas de manera que los estudiantes no perciben cuál es la relación de los contenidos dados en la escuela y la matemática de la vida cotidiana y en los primeros años de escolaridad es diferente.

Además, se debe tener en cuenta que si se pierde la confianza en la propia capacidad, pueden surgir sentimientos y emociones negativas que disminuyan el interés y la motivación por aprender (Mato, 2010). Seguidamente el estudiante entra en una dinámica de falta de implicación que repercute en su esfuerzo personal y, consecuentemente en el rendimiento.

Para terminar, y de cara al desarrollo de investigaciones futuras animamos a los profesores de matemáticas para que presten atención a las cuestiones afectivas y sus descriptores básicos como factores determinantes del rendimiento matemático y en la necesidad de abordar la evaluación del desarrollo socio-afectivo del alumnado, ya que sólo así será posible avanzar en una educación integral y completa (Murillo Torrecilla y Hernández Castillar, 2011).

Una propuesta por nuestra parte sería la de considerar el dominio afectivo en los planes de estudio de los maestros a la par que se trabaja el componente cognitivo. La realización de un programa de actuación no es aquí nuestro objetivo, pero sí queremos dejar patentes algunos aspectos que ese programa debería considerar: establecer principios de actuación que concreten las consideraciones pedagógicas y didácticas de las matemáticas y que gozan de una mayor utilidad, por lo que tienen de orientación específica (Mato, 2006).

También fomentar la colaboración de orientadores, tutores, investigadores ahondando en la afectividad de los docentes y, como dice Ferrando y Anguiano (2010) deben ser consideradas otras variables del ámbito del alumno. 


\section{BIBLIOGRAFÍA}

Abalde Paz, E., Barca Lozano, A., Muñoz Cantero, J. M., \& Ziemer, M. F. (2009). Rendimiento académico y enfoques de Aprendizaje: una aproximación a la realidad de la enseñanza superior brasileña en la región norte. Revista de Investigación Educativa, 27(2), 303-319.

Abramowicz, A., \& Moll, J. (2000). Para além do fracasso escolar. Campinas, Sao Paulo: Papirus.

Aliaga, J., \& Pecho J. (2000). Evaluación de la actitud hacia la Matemática en estudiantes secundarios. Revista Paradigmas, 1(1-2), 61-78.

Amorim, S. (2004). Improving student teachers' attitudes to Mathematics. En M. Johnsen and A. Berit (Eds.), The 28th Conference of the International Group for the Psychology of Mathematics Educat (Vol. 2, pp. 25-32). Bergen, Noruega: Universidad de Bergen.

Ashcraft, M. H. (2002). Math anxiety: Personal, educational and cognitive consequences. Current Directions in Psychological Sciences, 11(5), 181-185.

Bazán, J. L., \& Aparicio A. S. (2006). Las actitudes hacia la Matemática-Estadística dentro de un modelo de aprendizaje. Revista Semestral del Departamento de Educación, 25(28), 1-12.

Blanco, L. J. (2008). Una revisión crítica de la investigación sobre las actitudes de los estudiantes universitarios hacia la Estadística. Revista Complutense de Educación, 19(2), 311-330.

Broc Cavero, M. A. (2006). Motivación y rendimiento académico en alumnos de Educación Secundaria Obligatoria y Bachillerato LOGSE. Revista de Educación, 340, 379-414.

Callejo, M. (2004). Matemáticas para aprender a pensar. El papel de las creencias en la resolución de problemas. Madrid: Narcea.

Estrada, A. (2002). Análisis de las actitudes y conocimientos estadísticos elementales en la formación del profesorado (Tesis doctoral). Recuperado de http:// www.tesisde.com/t/ analisis-de-las-actitudes-y-conocimiento/10821/

Fennema, E., \& Sherman, J. (1976). Fennema-Sherman Mathematics Attitudes Scales: Instruments designed to measure attitudes toward the Llearning of mathematics by males and females. Catalog of Selected Documents in Psychology, 6(1), 31 (Ms. No. 1225).

Ferrando, P. J., \& Anguiano, C. (2010). El análisis factorial como técnica de investigación en Psicología. Papeles del Psicólogo, 3(1), 18-33.

Fierro-Hernández, C. (2006). Valoración del impacto de un programa de educación en valores en el último curso de Educación Secundaria Obligatoria. Revista de Educación, $339,455-466$.

Gil, N., Blanco, L., \& Guerrero, E. E. (2005). El papel de la afectividad en la resolución de problemas matemáticos. Revista de Educación, 340, 551-569.

Gómez-Chacón, M. (2000). Matemática emocional. Los afectos en el aprendizaje matemático. Madrid: Narcea.

Gómez-Chacón, I. M. (2002). Afecto y aprendizaje matemático: causas y consecuencias de la interacción emocional. En J. Carrillo (Coord.), Reflexiones sobre el pasado, presente y futuro de las matemáticas (pp. 197-227). Huelva: Publicaciones Universidad de Huelva.

Gómez-Chacón, I. M. (2006). Creencias de los estudiantes de matemáticas. La influencia del contexto de clase. Enseñanza de las Ciencias, 24, 309,324. 
Gómez-Chacón, I. M. (2007). Sistema de creencias sobre las matemáticas en alumnos de secundaria Creencias. Revista Complutense de Educación, 18, 125-143.

Hidalgo, A., Maroto, A., \& Palacios, A. (2005). El perfil emocional matemático como predictor de rechazo escolar: una relación con las destrezas y conocimientos desde una perspectiva evaluativa. Educación Matemática, 17, 86-116.

Kazelskis, R. (2000). Mathematics anxiety and test anxiety: Separate constructs? Journal of Experimental Education, 68(2), 137-146.

Martínez Padrón, O. (2005). El dominio afectivo en la Educación Matemática: Aspectos teóricosreferenciales a la luz de los Encuentro Edumáticos. Turmero, Venezuela: Universidad Pedagógica Experimental Libertador.

Mato, M. D. (2006). Diseño y validación de dos cuestionarios para evaluar las actitudes y la ansiedad hacia las matemáticas en alumnos de Educación Secundaria Obligatoria. (Tesis doctoral no publicada). Universidad de La Coruña, La Coruña (España).

Mato, M. D. (2010). Mejorar las actitudes hacia las matemáticas. Revista galego-portuguesa de psicoloxía e educación, 18(1), 1138-1663.

Mato, M. D., \& Muñoz, J. M. (2010). Evaluación de las actitudes hacia las matemáticas y el rendimiento académico. Revista de Ciencias Psicológicas, 4(1), 27-40.

Morales, P. (2006). Medición de las actitudes en Psicología y Educación. Construcción de cuestionarios y problemas metodológicos (3 $3^{\underline{a}}$ ed.). Madrid: Universidad Pontificia de Comillas.

Muñoz J.M., \& Mato, M. D. (2008). Análisis de las actitudes respecto a las matemáticas en alumnos de ESO. Revista de Investigación Educativa, 26(1), 209-226.

Murillo Torrecilla, F. J., \& Hernández Castilla, R. (2011). Efectos escolares de factores socio-afectivos. Un estudio multinivel para Iberoamérica. Revista de Investigación Educativa, 29(2), 407-427.

Peralbo, M., \& Barca, A. (2003). El fracaso escolar ¿cómo argumento? Revista GalegoPortuguesa de Psicoloxía e Educación, 7, 127-158.

Piaget, J. (1981). Intelligence and affectivity: Their relationship during child development. Annual Reviews Monograph. Palo Alto, California: Annual Reviews.

Torre, E., \& Mato, M. D. (2009). Ansiedade e rendemento en matemáticas. Revista Galega do Ensino, 56, 73-77.

Tyson, N. (2001). Fear of numbers. Natural History, 110(10), 30-32.

Watt, H. M. G. (2000). Measuring attitudinal change in mathematics and English over 1st year of junior high school: A multidimensional analysis. The Journal of Experimental Education, 68(4), 331- 361.

Zabalza, M. (1994). Evaluación de actitudes y valores. Evaluación del aprendizaje de los estudiantes. Barcelona: Graó.

Zan, R., Brown, L., Evanz, J., \& Hannula, M. S. (2006). Affect in mathematics education: an introduction. Educational Studies in Mathematics, 63, 113-121.

Fecha de recepción: 20 de diciembre de 2012.

Fecha de revisión: 20 de diciembre de 2012.

Fecha de aceptación: 27 de mayo de 2013. 